\title{
Atenção domiciliar no Sistema Único de Saúde: perfil de pacientes assistidos
}

\author{
Home care in the national health system: \\ profile of assisted patient
}

\author{
Derdried Athanasio Johann ${ }^{1}$, Clovis Cechinel $^{2}$, Talita Ferreira \\ Turatti do Carvalhal ${ }^{3}$, Marcelo Costa Benatto ${ }^{4}$, \\ Guilherme Parreira da Silva ${ }^{5}$ Joaninha Artigas de Lara ${ }^{6}$
}

\section{Resumo}

Objetivo: delinear o perfil dos pacientes assistidos em um Serviço de Atendimento Domiciliar da cidade de Curitiba. Métodos: desenho transversal, retrospectivo, abordagem quantitativa; coleta de dados dos pacientes assistidos no serviço em 2017; contendo as variáveis sociodemográficas e clínicas. Realizou-se análise estatística descritiva e modelos de regressão. Resultados: em 2017 houve 1798 atendimentos de 1436 pacientes. O perfil apontou maioria dos pacientes do sexo feminino, média de idade acima de 71 anos e raça branca; a procedência predominante dos assistidos foram as Unidades Básicas de Saúde ou Estratégia de Saúde da Família com tempo médio de atendimento superior a 38 dias; prevalência de distúrbios neurológicos; desfecho de atendimento foi alta. O custo médio mensal foi de R \$ 1.403,56 por paciente. Conclusão: o perfil dos pacientes indicou maioria de mulheres, brancas, com até oito anos de estudo, idade acima de 71 anos e assistidos por distúrbios neurológicos.

Palavras-chave: Serviços de assistência domiciliar. Perfil de saúde.

1 Doutorado em Enfermagem pela Universidade Federal do Paraná, Curitiba, Paraná, Brasil. Enfermeira do Espaço Saúde do Instituto Federal do Paraná, Curitiba, Paraná, Brasil.

2 Mestrado em Enfermagem pela Universidade Federal do Paraná, Curitiba, Paraná, Brasil. Coordenador médico do Programa Melhor em Casa Hospital do Idoso Zilda Arns, Curitiba, Paraná, Brasil. E-mail: cechinelc@hotmail.com

3 Especialização em Fisioterapia Cardiorrespiratória pela Faculdade Inspirar, Curitiba, Paraná, Brasil. Fisioterapeuta na Fundação Estatal de Atenção Especializada em Saúde de Curitiba, Curitiba, Paraná, Brasil.

4 Mestrado em Psicologia Clínica pela Universidade Federal do Paraná, Curitiba, Paraná, Brasil. Analista de Treinamento e Desenvolvimento na Fundação Estatal de Atenção Especializada em Saúde de Curitiba, Curitiba, Paraná, Brasil.

5 Graduação em Estatística, Curitiba, Paraná, Brasil.

6 Graduação em Enfermagem pela Pontifícia Universidade Católica, Curitiba, Paraná, Brasil. 


\begin{abstract}
Objective: to delineate the profile of patients assisted at a Home Care Service in the city of Curitiba. Methods: transversal design, retrospective, quantitative approach; data collection comprised all patients assisted at the site in 2017; sociodemographic and clinical variables. Descriptive statistical analysis and regression models were performed. Results: in 2017 there were 1798 visits of 1436 patients. The profile showed most of the female patients, mean age above 71 years and white race; the predominant provenance of the assisted ones were the Basic Health Units or Family Health Strategy with a mean attendance time of more than 38 days; prevalence of neurological disorders; treatment outcome was high. The average monthly cost was R $\$ 1,403.56$ per patient. Conclusion: the patients profile indicated a majority of white women, with up to eight years of schooling, age above 71 years old and assisted by neurological disorders.
\end{abstract}

Keywords: Home care services. Health profile.

\section{Introdução}

Os cuidados em saúde no Brasil têm sido evidenciados por meio da incorporação de tecnologias e terapêuticas inovadoras. Visando situações de dependência funcional, envelhecimento populacional e aumento de sobrevida de crianças e jovens portadores de condições crônicas complexas, nota-se demanda acentuada de cuidados e aporte de recursos ao Sistema Único de Saúde (SUS). ${ }^{(1)}$ No intuito de proporcionar qualidade de vida e otimização de recursos ao Estado, instituiu-se, de modo sistêmico, a Atenção Domiciliar (AD), no âmbito do SUS, com a criação do Programa Melhor em Casa (2011). Em Curitiba, o Serviço de Atenção Domiciliar (SAD) é gerido pela Fundação Estatal de Atenção Especializada em Saúde de Curitiba (FEAES), criada por meio da Lei Municipal 13.663 de 21 de dezembro de 2010.(2)

AAD é uma modalidade de cuidado integrada às Redes de Atenção à Saúde (RAS), caracterizada por um conjunto de ações de prevenção e tratamento de doenças, reabilitação, paliação e promoção à saúde, prestadas em domicílio. ${ }^{(3-4)}$ Atualmente é dividida em modalidades específicas de atendimento, a saber: AD1 - composta por usuários que requerem cuidados com menor frequência e necessidade de intervenções multiprofissionais, pressupondo estabilidade clínica e cuidados satisfatórios pelos cuidadores; o acompanhamento é de responsabilidade, conjunta e articulada, das equipes de Atenção Primária, por meio de visitas regulares em domicílio, no mínimo, uma vez por mês; AD2 - usuários com acompanhamento domiciliar para evitar ou abreviar uma hospitalização (em casos de agudizações de doenças crônicas, comprometimento severo de afecções crônicodegenerativas ou cuidados paliativos intensos); o acompanhamento é de responsabilidade do SAD, mas agregado à sua atuação permanece o cuidado longitudinal realizado pela Atenção Primária; e AD3 - atende usuários com condição clínica complexa, que requerem cuidado multiprofissional frequente, uso de equipamento(s) ou agregação de procedimento(s) de maior diversidade (ventilação mecânica, paracentese de repetição, nutrição parenteral e transfusão sanguínea); a responsabilidade do acompanhamento é do SAD e usualmente demanda períodos maiores de acompanhamento domiciliar. ${ }^{(1,3-4)}$

A AD não deve ser substitutiva à internação hospitalar, mas realizada quando as condições clínicas do usuário e da família permitirem, objetivando o conforto e humanização do cuidado à população. Assim, a $\mathrm{AD}$ configura-se como possibilidade de cuidado que promove maior bem-estar ao usuário e sua família e permite a continuidade do acompanhamento em saúde. ${ }^{(1)}$ O governo, em suas três esferas, visa reduzir os custos decorrentes da assistência hospitalar, bem 
como as próprias instituições de saúde. Com a implementação da AD, a despesa do cuidado é dividida com a família, além de estimular os vínculos entre os familiares e o paciente, no contexto domiciliar. ${ }^{(5)}$

O SAD objetiva a redução da demanda por atendimento hospitalar ou redução do período de permanência de usuários internados; a humanização da atenção à saúde, com a ampliação da autonomia dos usuários, a desinstitucionalização e a otimização dos recursos financeiros e estruturais. Organiza-se por meio das Equipes Multiprofissionais de Atenção Domiciliar (EMAD) e Equipes Multiprofissionais de Apoio (EMAP), atuando complementarmente aos cuidados da Atenção Primária, serviços de urgência e emergência e substitutivo ou suplementar à internação hospitalar. No ano de 2016, havia equipes em funcionamento em 25 estados; a disponibilidade de cuidado representou em média $26 \%$ da população brasileira e foram atendidos, em média, 34 mil pacientes por mês. ${ }^{(1)}$

Diante desse contexto, objetivou-se delinear o perfil dos pacientes assistidos em um Serviço de Atendimento Domiciliar (SAD) da cidade de Curitiba. Ao delinear o perfil da população atendida, pretende-se a melhoria do serviço, mediante a análise de dados que permita o planejamento adequado de assistência à saúde, minimizando os riscos e provendo a qualidade de vida.

\section{Metodologia}

Estudo transversal quantitativo de caráter retrospectivo no período de 2017, utilizando dados secundários de planilhas gerenciais e prontuário de pacientes acompanhados em um SAD de Curitiba. Optou-se pelo recorte temporal de 2017, para evidenciar a nova gestão municipal, o perfil dos pacientes atendidos e a necessidade de ajustes no processo de trabalho para atender as recomendações do Ministério da Saúde, que focaliza na desospitalização.

A coleta ocorreu em fevereiro de 2018, mediante dados do Sistema de Gestão Hospitalar
Philips Tazy ${ }^{\circledR}$, tabulados em planilhas eletrônicas do Microsoft Excel ${ }^{\circledR}$, e compôs-se das seguintes variáveis: idade, sexo, raça, religião, grau de instrução, data de atendimento inicial, data de alta ou óbito, procedência (instituição que determinou $\mathrm{o}$ atendimento pelo SAD), diagnóstico nosológico e equipe de atendimento. Categorizaram-se os diagnósticos nosológicos por sistemas corporais, conforme classificação do Código Internacional de Doenças, versão 10 (CID 10). Ressalta-se que algumas doenças recorrentes da amostra foram descritas sucintamente, na classificação por sistemas.

A análise estatística descritiva das variáveis numéricas deu-se por meio de medidas de tendência central, e para as variáveis nominais, tabelas de frequência absoluta e relativa. Considerando as variáveis de interesse, por conta de sua grandeza, executaram-se diferentes modelos: para frequência de atendimentos ( 1 a 5 ) ajustou-se um modelo de regressão ordinal; para as variáveis diagnóstico por sistema (neurológico/cardiológico/respiratório) e desfecho do atendimento (em atendimento, alta ou óbito) usou-se um modelo multinominal; o tempo de atendimento utilizou um modelo Tweedie. No intuito de avaliar as covariáveis idade, sexo e raça e a sua correlação com a variável de interesse, aplicou-se o Akaike Information Criteria (AIC). Para a variável frequência de atendimentos também foi considerado como covariável diagnóstico por sistema; e para tempo de atendimento o desfecho do atendimento (óbito/ alta). Utilizou-se o software R Core Team, versão 2017, para desenvolver as análises.

A análise do custo por paciente incluiu os custos mensais de transporte, insumos e medicamentos e despesas com a folha de pagamento. Assim, contabilizou-se o custo mensal do $\mathrm{SAD}$ e dividiu-se pelo número de pacientes atendidos no referido mês, calculando-se uma média de valores por paciente.

Declara-se não haver conflitos de interesse na execução da pesquisa, sendo aprovada pelo Comitê de Ética em Pesquisa, sob parecer número 1.826.246. 


\section{Resultados}

No ano de 2017 o SAD assistiu 1436 pacientes em 1798 atendimentos (média de 1,25 vez). O perfil sociodemográfico apontou maioria dos pacientes do sexo feminino $(58,6 \% ; n=841)$, religião não informada $(54,3 \% ; n=780)$, até oito anos de estudos $(56 \%$; $n=804)$, média de idade de 71,79 anos ( $\mathrm{SD} \pm 18,31$, máximo de 102 e mínimo de menor de um, mediana de 76) e raça branca (77,4\%; n=1111). Quanto aos dados clínicos, a frequência de atendimento variou de uma (80,8\%; $\mathrm{n}=1161)$ a cinco $(\mathrm{n}=1 ; 01 \%)$ vezes; a procedência do atendimento predominante foi da Atenção Primária em Saúde (APS) com 76\% (n=1367); o tempo médio de atendimento foi de 38,91 dias $(\mathrm{SD} \pm 35,51$, máximo de 325, mínimo de um e mediana de 30); o diagnóstico mais prevalente foi o distúrbio neurológico, com 369 ocorrências, sendo recorrente o acidente vascular cerebral e suas sequelas $(50,13 \%$; $\mathrm{n}=185$ ); e as altas foram o principal desfecho de atendimento no $\operatorname{SAD}(77,7 \% ; n=1397)$ (Tabela 1$)$.

Tabela 1 - Distribuição dos pacientes segundo características sociodemográficas e clínicas. Curitiba-PR, 2017. ( $\mathrm{n}=1436)$.

\begin{tabular}{|c|c|}
\hline Variáveis & n $(\%)$ \\
\hline \multicolumn{2}{|c|}{ Características sociodemográficas } \\
\hline Idade & $\mathrm{X}=71,79(\mathrm{SD} \pm 18,31)^{*}$ \\
\hline \multicolumn{2}{|l|}{ Sexo } \\
\hline Feminino & $841(58,6)$ \\
\hline Masculino & $595(41,4)$ \\
\hline \multicolumn{2}{|l|}{ Religião } \\
\hline Católica & $441(30,7)$ \\
\hline Outras & $200(13,9)$ \\
\hline Sem religião & $15(1,1)$ \\
\hline Não informado & $780(54,3)$ \\
\hline \multicolumn{2}{|l|}{ Anos de estudo } \\
\hline Sem instrução & $215(15)$ \\
\hline Até 8 anos & $804(56)$ \\
\hline De 8 a 11 anos & $156(10,9)$ \\
\hline 12 anos ou mais & $58(4)$ \\
\hline \multicolumn{2}{|l|}{ Raça } \\
\hline Branco & $1111(77,4)$ \\
\hline Negro ou pardo & $147(10,2)$ \\
\hline Amarelo ou indígena & $10(0,7)$ \\
\hline Não informado & $168(11,7)$ \\
\hline \multicolumn{2}{|c|}{ Características clínicas } \\
\hline \multicolumn{2}{|l|}{ Frequência de atendimento } \\
\hline 1 & $1161(80,8)$ \\
\hline 2 & $205(14,3)$ \\
\hline 3 & $54(3,8)$ \\
\hline 4 a 5 & $16(1,1)$ \\
\hline
\end{tabular}


Continuação

Procedência

UBS/ESF

1367 (76)

Hospitais

$255(14,2)$

UPA

$158(8,8)$

Outros

$18(1)$

\section{Características clínicas}

Tempo de atendimento

$\mathrm{X}=38,91(\mathrm{SD} \pm 35,51)^{*}$

Diagnóstico nosológico

Distúrbios cardiológicos e cardiovasculares

$93(100)$

Distúrbios dermatológicos

$121(100)$

Geral

$26(21,49)$

Úlcera por pressão

$95(78,51)$

Distúrbios endocrinológicos

$19(100)$

Distúrbios gastrointestinais

$36(100)$

Distúrbios geniturinários

$107(100)$

Distúrbios hematológicos ou imunológicos

$6(100)$

Distúrbios hematológicos

$2(33,33)$

Distúrbios imunológicos

$4(66,67)$

Distúrbios musculoesqueléticos

$72(100)$

Geral

$26(36,11)$

Trauma

$46(63,89)$

Distúrbios neurológicos

$369(100)$

Acidente vascular cerebral e suas sequelas

$185(50,13)$

Alzheimer

$32(8,67)$

Demência

$103(27,91)$

Outros

$49(13,28)$

Distúrbios nutricionais e/ou metabólicos

$8(100)$

Distúrbios psicológicos

$16(100)$

Distúrbios respiratórios

$183(100)$

Doença pulmonar obstrutiva crônica (DPOC)

$63(34,43)$

Geral

$120(65,58)$

Neoplasia

$52(100)$

Cuidados paliativos

$10(100)$

Outros

$32(100)$

Outras infecções (virais, bacterianas e septicemia)

$15(46,88)$

Outros

$17(53,12)$

Desfecho do atendimento
Alta
$1397(77,7)$
Em acompanhamento
$128(7,1)$
Óbito
$273(15,2)$

Fonte: Autores

* $\mathrm{X}=$ média e $\mathrm{SD}=$ desvio padrão. 
As inferências estatísticas analisadas referem-se às variáveis: frequência de atendimento, diagnóstico nosológico (categorizados por sistemas) tempo e desfecho de atendimento. Para a variável frequência de atendimento (categorias de uma a cinco vezes), a distribuição da idade e sexo foi similar entre as categorias da variável resposta. Destacam-se idades mais elevadas na frequência de atendimento de quatro vezes, com média de idade de 82,09 anos ( $\mathrm{SD} \pm 7,92$, mediana 81, mínimo 67 e máximo 100 anos). Sugere-se, ao compararmos pacientes de 80 anos de idade com outros de 79, uma tendência estatística para frequências de atendimentos superiores $(1,009$ vez; $\mathrm{p}=0,072$ ). Para diagnósticos por sistema, os dados sugerem uma proporção elevada de distúrbios cardiológicos ou cardiovasculares em relação aos neurológicos e respiratórios $(79,2 \%$, $63,9 \%$ e $64,2 \%$ respectivamente) para um único atendimento, totalizando $65,9 \%$. Os distúrbios neurológicos e respiratórios aumentaram as chances de maior frequência de assistência (2,27 e 2,08 vezes respectivamente, valores de $\mathrm{p}=0,007$ e 0,03 ) em relação aos distúrbios cardiológicos (Tabela 2).

Com relação à variável diagnóstico nosológico, considerou-se para as análises os mais frequentes, a saber: distúrbios cardiológicos ou cardiovasculares, respiratórios e neurológicos. Os dados gerais apontaram uma igualdade na distribuição das idades referente aos distúrbios. A raça predominante nos três distúrbios foi branca. Destaca-se o diagnóstico de distúrbios neurológicos no sexo masculino $(64 \% ; n=188)$. O modelo de regressão, para a variável diagnóstico nosológico, foi significativo nas comparações: distúrbios respiratórios têm mais chances de serem desenvolvidos por sexo feminino do que os neurológicos em 1,54 vez $(\mathrm{p}=0,0141)$, bem como por outras raças (em relação à branca) em 2,02 vezes $(p=0,0046)$ (Tabela 2$)$.

O tempo de atendimento para as variáveis raça e sexo foi semelhante; com relação à idade a relação foi nula (apontado pelo coeficiente de correlação linear de Pearson -0,0008). No modelo de regressão, a chance de o paciente ter maior tempo de atendimento foi de 1.219 vezes $(\mathrm{p}=0,0014)$ quando obteve alta se comparado ao óbito (Tabela 2).

O desfecho do atendimento obteve idades superiores para pacientes que foram a óbito, sendo média de 79,41 anos ( $\mathrm{SD} \pm 12,2$, mediana 81, mínimo 16 e máximo 102 anos). A análise da regressão multinomial apontou que o aumento em uma unidade para a variável idade elevou $(1,01$ vez; $p=0,002)$ as chances de o paciente permanecer em acompanhamento em relação a receber alta do $\mathrm{SAD}$; a relação entre óbito e alta do atendimento aponta o aumento em 1,06 a chance do óbito $(\mathrm{p}<0,0001)$; bem como aumenta em 1,04 vez a chance de o paciente vir a óbito em relação ao paciente que permaneceu em acompanhamento $(p<0,0001)$. Se o paciente fosse do sexo masculino, sua chance de vir a óbito, comparado a obter alta, foi de 1,90 vez maior que do sexo feminino $(\mathrm{p}=0,004)$; assim como a chance de vir a óbito em relação a estar em acompanhamento foi de 1,77 vez maior que do sexo feminino $(\mathrm{p}<0,0001)$ (Tabela 2$)$. 
Tabela 2 - Resultados principais do ajuste de modelos de regressão.

\begin{tabular}{|c|c|c|c|c|c|}
\hline Variável & Estimativa & Erro padrão & $\begin{array}{l}\text { Razão de } \\
\text { chances }\end{array}$ & IC $[95 \%]$ & Valor -p \\
\hline \multicolumn{6}{|l|}{ Frequência de atendimento } \\
\hline $\begin{array}{l}\text { Distúrbios neurológicos x } \\
\text { cardiológicos ou cardiovasculares }\end{array}$ & 0,818 & 0,27 & 2,27 & 1,$203 ; 4,273$ & $0,002 *$ \\
\hline $\begin{array}{l}\text { Distúrbios respiratórios x } \\
\text { cardiológicos ou cardiovasculares }\end{array}$ & 0,732 & 0,287 & 2,08 & 1,$060 ; 4,082$ & $0,029 *$ \\
\hline $\begin{array}{l}\text { Distúrbios neurológicos } \mathrm{x} \\
\text { respiratórios }\end{array}$ & 0,085 & 0,172 & 1,09 & 0,$728 ; 1,631$ & $0,873^{*}$ \\
\hline Idade & 0,009 & 0,005 & 1,01 & 0,$999 ; 1,019$ & 0,072 \\
\hline \multicolumn{6}{|l|}{ Diagnóstico nosológico } \\
\hline $\begin{array}{l}\text { Feminino x masculino / } \\
\text { distúrbios cardiológicos ou } \\
\text { cardiovasculares x neurológicos }\end{array}$ & 0,403 & 0,236 & 1,50 & 0,$942 ; 2,377$ & 0,088 \\
\hline $\begin{array}{l}\text { Feminino x masculino / } \\
\text { distúrbios respiratórios x } \\
\text { neurológicos }\end{array}$ & 0,431 & 0,175 & 1,54 & 1,$091 ; 2,171$ & 0,014 \\
\hline $\begin{array}{l}\text { Outras raças x branca / distúrbios } \\
\text { respiratórios x neurológicos }\end{array}$ & 0,705 & 0,249 & 2,02 & 1,$244 ; 3,300$ & 0,005 \\
\hline \multicolumn{6}{|l|}{ Tempo de atendimento } \\
\hline Alta x óbito & 0,198 & 0,062 & 1,22 & 1,$080 ; 1,376$ & 0,001 \\
\hline \multicolumn{6}{|l|}{ Desfecho do atendimento } \\
\hline $\begin{array}{l}\text { Idade / em acompanhamento } \mathrm{x} \\
\text { alta }\end{array}$ & 0,014 & 0,004 & 1,01 & 1,$005 ; 1,023$ & 0,002 \\
\hline Idade / óbito x alta & 0,055 & 0,007 & 1,06 & 1,$042 ; 1,071$ & $<0,0001$ \\
\hline $\begin{array}{l}\text { Idade / óbito } \mathrm{x} \\
\text { em acompanhamento }\end{array}$ & 0,041 & 0,005 & 1,04 & 1,$031 ; 1,053$ & $<0,0001$ \\
\hline $\begin{array}{l}\text { Masculino x feminino / } \\
\text { óbito } \mathrm{x} \text { alta }\end{array}$ & 0,644 & 0,227 & 1,90 & 1,$221 ; 2,969$ & 0,004 \\
\hline $\begin{array}{l}\text { Masculino x feminino / } \\
\text { óbito x em acompanhamento }\end{array}$ & 0,571 & 0,139 & 1,77 & 1,$347 ; 2,325$ & $<0,0001$ \\
\hline
\end{tabular}

Fonte: Autores

*Valor -p com correção de Tukey (devido a comparações múltiplas).

Os custos relativos ao atendimento do SAD no ano de 2017, os quais referem-se às despesas com folha de pagamento, transporte das equipes de atendimento, insumos e medicamentos utilizados nos cuidados prestados aos pacientes em atenção domiciliar, foram de $\mathrm{R} \$ 6.690 .341,26$. $\mathrm{O}$ custo mensal por paciente foi em média de
$\mathrm{R} \$ 1.403,56$ ( $\mathrm{SD} \pm 103,33$, máximo $\mathrm{R} \$ 1.603,63$ mínimo R\$ 1.243,08, mediana R\$ 1.400,89). Destaca-se que os 1436 pacientes atendidos no SAD foram assistidos por 10 equipes EMAD tipo 1 e três EMAP. O repasse financeiro anual, calculado pelo número de equipes cadastradas no Ministério da Saúde, totalizou R\$ 6.216.000,00. 


\section{Discussão}

As limitações da pesquisa relacionam-se ao desenho da pesquisa transversal, de análise de dados retrospectivos, os quais não puderam ser controlados, mediante o fato do preenchimento do banco de dados ser realizado por diversas categorias profissionais, impossibilitando uma padronização prévia. O prontuário do paciente precisa transpor o papel de registro de informações para um instrumento de comunicação, maximizando o tratamento e conforto do paciente. ${ }^{(6)}$

Ao delinear o perfil da população atendida, analisar e apresentar dados sobre a caracterização sociodemográfica e clínica dos pacientes assistidos, permite-se a fundamentação de evidências e o planejamento adequado do cuidado em saúde, visando prática clínica segura, minimização de riscos, melhoria contínua do serviço e qualidade de vida ao paciente no domicílio.

Os resultados desta pesquisa destacam 1436 pacientes assistidos sob 1798 atendimentos do SAD, pelo SUS. Estudos apontaram valores inferiores no mesmo período de acompanhamento, de um ano, com 438 (ano de 2011) e 388 (2012) pacientes analisados, ${ }^{(7)}$ enquanto que em rede privada de saúde, no ano de 2013, atendeu-se no domicílio um total de 2943 pacientes. ${ }^{(8)}$ A nível nacional, um estudo epidemiológico identificou a prevalência de $11,7 \%$ (IC 95\% 10,9;12,5) de atenção domiciliar. ${ }^{(9)}$

Apontou-se predominância de mulheres assistidas no domicílio por equipes de AD. Estudos nacionais apontam característica semelhante em SAD estudados no País, como maioria de pacientes sendo mulheres com valores de $60 \%{ }^{(5)}$ e acima de $53 \%{ }^{(7)}$ e $72 \%$. $^{(10)}$ Ao referir-se às mulheres em maior composição na amostra, ressaltou-se que comparado aos homens, estes têm chance de 1,90 vez maior de vir a óbito quando comparado à alta do atendimento. Ou seja, mesmo permanecendo em maiores proporções de atendimento, as mulheres têm mais chances de receber alta. Dos 1436 pacientes, $273(15,2 \%)$ vieram a falecer no ano de acompanhamento. Semelhante aos achados de um estudo internacional, no qual 290 (28,9\%; $\mathrm{n}=1001$ ) morreram durante o período de um ano e, na análise de regressão logística, as mulheres apresentaram menor risco de morte $[\mathrm{OR}=0,67$ $(0,50-0,91)] .{ }^{(11)}$ Um estudo de prevalência de $\mathrm{AD}$, após análise ajustada, apontou probabilidade das mulheres receberem atendimento domiciliar 1,4 vez maior quando comparada aos homens. ${ }^{(8)}$

As médias de idade encontradas para a população total analisada foram superiores a 71 anos; nos pacientes que evoluíram para óbito durante o atendimento as médias superam os 79 anos de idade. Estudo realizado em 2016 apontou média de idade superior de 81,6 anos; ${ }^{(10)}$ médias de idade inferiores das encontradas nessa pesquisa perfizeram $62,66 \pm 18,31$ anos, sendo que 50,6\% da amostra apresentaram idade acima dos 65 anos em outro estudo nacional.(7) Em relação à idade, concluiu-se aumento linear da prevalência de $\mathrm{AD}$, sendo a probabilidade $1,85 \mathrm{vez}$ maior para idosos com mais de 80 anos quando comparados ao grupo de 60 a 64 anos. ${ }^{(8)}$

Divergente aos achados dessa pesquisa, na qual se sugere uma necessidade maior de atendimentos ou até mesmo o óbito do paciente com o avanço da idade, estudo apontou menores intervenções com a progressão positiva da idade, apontando a necessidade de novos estudos relacionando idade e atenção domiciliar. ${ }^{(7)}$ Projeções relacionadas ao envelhecimento indicam que haverá mais idosos em 2050 do que crianças; em 2012, 12,8\% da população brasileira era composta por idosos, totalizando cerca de 19,6 milhões de pessoas, e esse número deve ultrapassar os 41,5 milhões até 2030. ${ }^{(12-14)}$ No Paraná, a expectativa de vida ao nascer ultrapassará os 80 anos até 2030; em 2012, 11\% da população paranaense era composta por pessoas com idade igual ou superior a 60 anos. ${ }^{(13)}$ Manejar a saúde do idoso, mediante alternativas humanizadas, personalizadas e no domicílio, é desafiador, mas uma saída perspicaz para a desospitalização e cuidado ao idoso, focado na redução da demanda de internações hospitalares e otimização dos custos desse atendimento, bem como na formação dos profissionais. ${ }^{(3,5)}$ 
Com relação à raça, estudo realizado em 100 municípios brasileiros, com residentes em zona urbana, apontou prevalência da cor da pele (autorreferida) mestiça (52,9\%), seguida da branca $(40,3 \%),{ }^{(9)}$ contrapondo os encontrados desta pesquisa, de maioria branca. Ressalta-se que a presente pesquisa foi realizada na Região Sul do País, a qual é de colonização européia (alemães, italianos, poloneses, ucranianos, entre outros), em sua grande maioria, o que justificaria tal achado. A maior parte dos pacientes assistidos no SAD possuía até oito anos de estudo, similar ao apresentado por estudo em $45,3 \%$ da população (de um a sete anos de estudo), bem como não ter nenhum ano de estudo aumentou em 2,2 vezes a probabilidade de recebimento de atenção domiciliar, quando comparados com idosos que tinham oito anos ou mais de estudos. ${ }^{(9)}$

A procedência predominante dos pacientes assistidos no SAD foi de UBS/ESF. Destacase que houve encaminhamentos também de Unidades de Pronto Atendimento e instituições hospitalares. O SAD, sendo constituído por equipe multiprofissional especializada, possibilita um elo de cuidado entre o hospital e a rede básica, ou apresenta caráter substitutivo às internações de baixa/média complexidade. Assim, trata-se de uma potente "porta de saída" junto aos serviços de urgência/emergência, evitando internações hospitalares desnecessárias. ${ }^{(1)}$ Estudo concluiu procedência equivalente aos achados dessa pesquisa, sendo $60,40 \%$ pacientes advindos de UBS/ESF e $27,60 \%$ de unidades de saúde geridas pela instituição hospitalar de referência. ${ }^{(7)}$

Os pacientes assistidos pelo $\mathrm{SAD}$, no ano de 2017, tiveram um tempo de atendimento superior a 38 dias (máximo de 325 dias). Média de atendimento de 21,5 dias (13-36) foi caracterizada por estudo no extremo sul do País. ${ }^{(7)}$ Relacionado ao desfecho do atendimento domiciliar, a maioria dos pacientes obteve alta, assim como em 76\% dos casos de estudo semelhante ${ }^{(7)}$. Óbitos tiveram taxas de $1 \%{ }^{(8)}$ e $2,7 \%{ }^{(7)}$ dos pacientes assistidos, dados inferiores ao achados dessa pesquisa.
Diagnóstico de distúrbios neurológicos (sendo prevalente o AVC e suas sequelas) predominou no sexo masculino, aumentando as chances para maior frequência de atendimentos, enquanto que os distúrbios respiratórios, quando comparados aos neurológicos, apresentaram maior chance de desenvolver-se em mulheres de outras raças (exceto a branca). Estudo retrata as causas mais frequentes de atendimento domiciliar: ocasionadas por pneumonia (26\%); infecção do trato urinário (8\%); insuficiência respiratória (5\%); acidente vascular encefálico (4\%); insuficiência cardíaca (4\%); fratura de fêmur (3\%); hemorragia digestiva (3\%); e depleção de volume $(3 \%){ }^{(8)}$

$\mathrm{O}$ custo do atendimento médio mensal foi de $\mathrm{R} \$ 1.403,56$, enquanto que o custo bruto anual foi $\mathrm{R} \$ 6.690 .341,26$. No Chile, o valor conjunto de 12 programas de um Sistema de Atenção Domiciliária variou entre $\$ 1.693,00$ a $\$ 6.328,00$ dólares mensais; já o custo bruto do sistema, no ano de 2012, superou \$ 1,214 milhão de dólares. ${ }^{(15)}$ O SAD é constituído por três EMAP e 10 EMAD do tipo 1; o financiamento, previsto em lei, no País, revela um incentivo financeiro de custeio mensal de 50 mil reais para EMAD tipo 1, 34 mil reais para EMAD tipo 2 e seis mil reais para cada EMAP. $^{(3,16)}$ Assim, percebe-se o subfinanciamento existente aos atuais gastos com o $\mathrm{SAD}$, dado que o custo total disponibilizado pelo Ministério da Saúde correspondeu a $92,91 \%$ do bruto anual calculado pelo número de pacientes assistidos. Na Sérvia, uma análise econômica sobre atenção domiciliar indicou a necessidade de, além do custeio do serviço, dois cenários prioritários para investimento, a fim de complementar e qualificar a assistência; são eles: aumentar o número de horas de atendimento domiciliar de seis para oito horas semanais, investindo primordialmente em recursos humanos, bem como incluir o maior número de beneficiários possíveis (chegando a $33 \%$ da população), gastando recursos de forma racional e proporcionando qualidade de vida aos pacientes. ${ }^{(17)}$ 


\section{Conclusões}

O perfil dos pacientes indicou maioria de mulheres, brancas, com até oito anos de estudo, idade acima de 70 anos, assistidos por distúrbios neurológicos e procedentes de UBS/ESF. Houve relações significativas entre a frequência de atendimento e sexo quando comparados ao diagnóstico nosológico; tempo de atendimento relacionado ao seu desfecho; e desfecho do atendimento relacionado à idade e sexo. $\mathrm{O}$ custo médio mensal de atendimento foi de $\mathrm{R} \$ 1.403,56$ por paciente.

Ao delinear o perfil da população atendida, assim como os custos assistenciais, permite-se um planejamento adequado do serviço e do cuidado em saúde, visando prática clínica integral e melhor aproveitamento do potencial de desospitalização do $\mathrm{SAD}$, culminando na otimização dos recursos da RAS do município e maior qualidade de vida dos pacientes em domicilio.

\section{Referências}

1 Ministério da Saúde. Programa de Desenvolvimento Institucional do Sistema Único de Saúde - PROADI - SUS. Projeto Complexidade do Cuidado na Atenção Domiciliar. Brasília (DF): Ministério da Saúde; 2017.

2 Curitiba. Lei Municipal 13.663 de 21 de dezembro de 2010. Institui a Fundação Estatal de Atenção Especializada em Saúde de Curitiba (FEAESCURITIBA), altera a Lei Municipal $n^{0} 7.671$, de 10 de junho de 1991, e dá outras providências. Curitiba -PR: Prefeitura Municipal; 2010.

3 Brasil. Ministério da Saúde. Portaria de Consolidação $n^{\circ}$ 5, de 28 de setembro de 2017. Consolidação das normas sobre as ações e os serviços de saúde do Sistema Único de Saúde. Brasília (DF): Ministério da Saúde; 2017.

4 Ministério da Saúde. Comissão Nacional de Incorporação de Tecnologia no SUS (CONITEC). Brasília (DF): Ministério da Saúde; 2013.
5 Bajotto AP, Witter A, Mahmud SJ, Sirena S, Goldim JR. Perfil do paciente idoso atendido por um programa de atenção domiciliar do Sistema Único de Saúde em Porto Alegre, RS. Rev HCPA. 2012;32(3):311-7.

6 Cavalheiro TB, Gouvea PB, Acosta AS, Maia SC, Grando SR, Rangel RCT. Registros da equipe multiprofissional sobre o acompanhamento de pacientes em estágio avançado de doença oncológica. Semina. 2017;38(2):175-84.

7 Machado DO, Silva FM, Mahmud SJ, Fengler FL, Paskulin LMG. Care demands regarding home-care service: a descriptive study. Braz J Nurs. 2014;13(3):353-61.

8 Biscione FM, Szuster DAC, Drumond EF, Ferreira GUA, Turci MA, Lima JFL Jr, et al. Avaliação de efetividade da atenção domiciliar de uma cooperativa médica de Belo Horizonte, Minas Gerais, Brasil. Cad. Saúde Pública. 2013; 29(Sup):S73-S80.

9 Wachs LS, Nunes BP, Soares UM, Facchini LA, Thumé E. Prevalência da assistência domiciliar prestada à população idosa brasileira e fatores associados. Cad. Saúde Pública. 2016;32(3):e00048515.

10 Prado FG, Rodrigues BB, Abud VRSP, Fajardo HSR, Bastos RMR. Perfil sociodemográfico e clínico da população idosa assistida pelo serviço de atendimento domiciliar de Juiz de Fora. Rev. APS. 2016;19(2):351-2.

11 Badia JG, Santos AB, Segura JCC, Puelles PG. Predictors of mortality among elderly dependent home care patients. BMC Health Services Research. 2013;13:316-23.

12 Lima CMB, Alves HVD, Mograbi DC, Pereira FF, Fernandez JL, Charchat-Fichman H. Performance on cognitive tests, instrumental activities of daily living and depressive symptoms of a community-based sample of elderly adults in Rio de Janeiro, Brazil. Dement. Neuropsychol. 2017;11(1):54-81.

13 Instituto Brasileiro de Geografia e Estatística 2013. Perfil dos Estados: Paraná. Brasília: IBGE; 2013. 
14 Fundo de Populações das Nações Unidas. Envelhecimento no Século XXI: Celebração e Desafios. Nova York: UNFPA; 2012.

15 Matus-López M, Pedraza CC. Costo de un sistema de atención de adultos mayores dependientes en Chile, 2012-2020. Rev Panam Salud Publica. 2014;36(1):31-6.

16 Brasil. Ministério da Saúde. Portaria $n^{\circ} 825$, de 25 de abril de 2016. Redefine a Atenção Domiciliar no âmbito do Sistema Único de Saúde (SUS) e atualiza as equipes habilitadas. Brasília: Ministério da Saúde; 2016.

17 Mihic M, Todorovic M, Obradovic V, Mitrovic Z. Can we do better? Economic analysis of human resource investment to improve home care service for the elderly in Serbia. Clin Interv Aging. 2016;11:85-96. 
